\title{
Head Pose Estimation Based On Detecting Facial Features
}

\author{
Hiyam Hatem ${ }^{1,3}$, Zou Beiji ${ }^{1}$, Raed Majeed ${ }^{1}$, Jumana Waleed ${ }^{1}$ and Mohammed \\ Lutf $^{2}$ \\ ${ }^{1}$ School of Information Science and Engineering, Central South University, \\ Changsha, 410083, China \\ ${ }^{2}$ Department of Electronics and information Engineering, Huazhong University of \\ science and technology, Wuhan, China \\ ${ }^{3}$ Department of Computer Science, Collage of Sciences, Baghdad University, Iraq \\ hiamhatim2005@yahoo.com,bjzou@vip.163.com,raed.m.muttasher@gmail.com, \\ jumana_waleed@yahoo.com,mohammed.lutf@gmail.com
}

\begin{abstract}
Head pose estimation is recently a more popular area of research. Challenging conditions, such as extreme pose, lighting, and occlusion, has historically hampered traditional, model-based methods. This paper presents a proposal of an integrated method for head pose estimation based on face detection and tracking. This method first locates certain facial features and based on their relative locations determine the head pose, the head pose estimated using coordinates of both eyes and a mouth relative to the nose as the origin of the coordinate system. The nose position is set up as the origin. The coordinates of the other parts defined from the origin, the distance between the face parts normalized so that the coordinates are independent of the image size. For facial feature detection from the detected face region, Haar-like feature utilized along with AdaBoost learning, the Adaboost learning algorithm used for creating optimized learning data.

From the experiments, the proposed approach shows robustness in face and facial feature detection and eventually produces better results in estimating head pose rather than simply using Haar-like feature for both face and facial feature detection. The computational cost is low because it uses only those three points.
\end{abstract}

Keywords: Face detection, Head-pose estimation, Geometric feature, AdaBoost learning function

\section{Introduction}

Estimating head pose correctly from input face images is important for developing applications of vision-based human computer interaction. Head pose estimation is a fundamental task for face recognition algorithms, ambient intelligence applications, and video-surveillance systems working in uncontrolled environments [1].

The development of smart phones, tablets and other mobile consumer electronics presents a large area for various applications of head pose tracking algorithms. Computer vision researchers have been exploring the topic of head pose estimation for the last 20 years. A lot of improvement has made over the years, but still no single method is capable of estimating the head pose both accurately and robustly in all situations. There are generally two categories of head pose estimation algorithms: coarse head pose estimation, which tend is more robust, but not necessarily accurate and fine head posing estimation, which demonstrate high accuracy [2]. Accurately localizing the head and its orientation is either the explicit goal of systems like human computer interfaces or a necessary preprocessing step for further analysis, such as identification or facial expression recognition. 
From the point of view of image representation, head pose estimation can categorize into two types: appearance-based methods and geometric-feature based methods. They can classify by the characteristics of the description vectors that are used to train; the appearance-based approach uses texture information from a facial image, whereas the geometric-feature-based approach manipulates positional information about the facial features such as eyes, eyebrows, nose, and mouth. The second method finds facial features using model-based algorithms such as the Active Shape Model (ASM), the Active Appearance Model (AAM), or the Constrained Local Model (CLM). Feature vectors can be composed of location information obtained using several facial feature detectors trained with another training set for 2D location detection [3]. The feature vectors, which are composed of the positional information, can be used as a supervised learning framework. Furthermore, they can be employed directly to estimate facial poses.

In the feature-based methods, the head pose inferred from the extracted features, which include the common feature visible in all poses, the pose-dependent feature, and the discriminated feature together with the appearance information. Vatahska et al., [4] Use a face detector roughly classifies the pose as frontal, left, or right profile. After his, they detect the eyes and nose tip using AdaBoost classifiers, and the detections fed into a neural network that estimates the head orientation.

Whitehill et al., [5] Present a discriminative approach to frame-by-frame head pose estimation. Their algorithm relies on the detection of the nose tip and both eyes, thereby limiting the recognizable poses to the ones where both eyes are visible. Morency et al., [6] Propose a probabilistic framework called Generalized Adaptive View-based Appearance Model integrating frame-by-frame head pose estimation, differential registration, and key frame tracking.

In the feature-based methods, the head pose inferred from the extracted features, which include the common feature visible in all poses, the pose-dependent feature, and the discriminate feature together with the appearance information.

Our approach in this paper is presents a proposal of an integrated method for face detection, tracking, and head pose estimation. The head poses estimated using coordinates of both eyes and a mouth relative to the nose as the origin of the coordinate system. The head poses estimated using coordinates of a left eye, a right eye, and a mouth relative to a nose, which is the origin of the coordinate system. Their coordinates obtained using face part tracking. The computational cost using only these three points is lower than the method using a complicated 3D head model.

\section{Geometric Approaches}

Humans can recognize head poses by detecting simple sets of edges, similar to cartoon faces. People are capable of identifying simple head poses because they can abstract the features of faces intuitively. In particular, people innately recognize the shapes, configurations, or contours of trained features such as eyes, noses, mouths, eyebrows, foreheads, and chins. Thus, people can remember abstracted images of heads by inference from trained data [7].

Geometric approaches derive the head pose from the geometric configuration of facial features depending on how the face positioned in relation to the silhouette of the head or how big the nose deviation is from bilateral symmetry.

One group of approaches uses the outer corners of the eyes, the outer corners of the mouth and the nose position [8]. The center points between the eye corners and between the lip corners project a line. The distance of the nose from this line, as well as the angle, gives information about the pose.

Geometric approaches usually require few calculations and are simple. They are also capable of providing a highly accurate estimation, but their accuracy strongly depends on the accuracy of facial feature location estimation. At present, the most accurate detectors 
have an error of at least 1-2 pixels even with high quality images. This is a lower bound on the potential accuracy of geometric methods [2]. In our experience, the 2-pixel feature location error can cause a considerably large, 5-10 degree head pose angle estimation error for typical webcam face images. It means that when using a simple webcam, geometric methods are unable to provide accurate head pose estimation because of limited feature detection accuracy. A further limitation of these methods is the ability to estimate the head pose only when all the facial features are visible and detectable.

\subsection{Facial Feature Detection Using Haar-like Feature}

The face is an important part of human anatomy that varies from individual to individual, but can be located with the use of certain context information. Facial feature detection methods classified into two: local and global methods. In local method, each face component like eyes, mouth and nose detected separately while in global method all facial parts detected jointly and a model based on the relative position of these features is constructed.

To estimate head pose from an input image, the detection of specific facial features is important. Because, in this work the locations of both eyes and nose used to estimate head pose. We use Haar-like feature and Adaboost learning algorithm to detect such facial features rather using pixel directly. Haar-like feature originated from the Haar basis functions that have been used by Papageorgiou et al., [9].

A Haar-like feature takes into account adjacent rectangular regions at a specific location in a window, sums up the pixel intensities in each region and calculates the difference between these sums. Subsequently the difference used to categorize the subsections of an image, Haar-like features can capture the intensity gradient at different locations, spatial frequencies and directions. The value of simple rectangle Haar-like feature defined as the difference of the sum of pixels of areas inside the rectangle, which can be at any position and scale within the detected face image, this rectangle feature can be computed very rapidly by using summed area table called integral image [10].

The possible wavelets in the candidate area are vastly numerous. It is inefficient to produce a classifier using all possible wavelets as features. Selecting effective features for detection is necessary. Haar-like features, computed according to the following equation:

$$
i i(x, y)-\sum_{x^{\prime} \leq x y \leq y} i\left(x^{x}, y^{\prime}\right)
$$

Where $\operatorname{ii}(x, y)$ is the integral image at pixel location $(x, y)$, and $i\left(x^{\prime}, y^{\prime}\right)$ is the original image. Calculation of the sum of a rectangular area in the original image is extremely efficient, requiring only four additions for any arbitrary rectangular size.The calculated feature values used for Adaboost classifier to recognize facial features such as eyes and nose. It can calculate using the following equation:

$$
\sum_{L x y, \in A B C D} \mathrm{ii}(\mathrm{x}, \mathrm{y})-\mathrm{ii}(\mathrm{A})+\mathrm{H}(\mathrm{D}) \quad \mathrm{ii}(\mathrm{B}) \quad \mathrm{in}(\mathrm{C})
$$

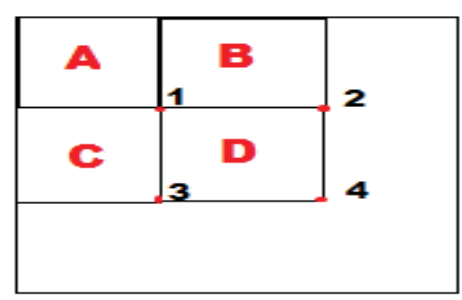

\footnotetext{
Figure 1. Integral Image at Location 1 is the Sum of Pixels in Region A; at Location 2 Sum of Pixels in Region $A+B$, at Location $3 \mathrm{C}+\mathrm{A}$ and at Location $4 \mathrm{~A}+\mathrm{B}+\mathrm{C}+\mathrm{D}$
} 
In our approach, we adopt nine generalized Haar-like features, including a group of extended Haar-like features proposed in [11] and 4 basic Haar-like features, to increase the detector's performance. Figure 2 compares the four basic Haar-like features applied by Viola and Jones as well as the generalized Haar-like features used in our approach.

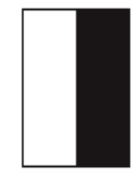

(1)

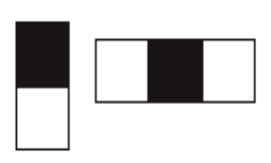

(3)

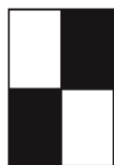

(4)

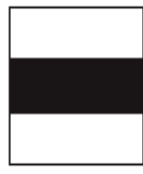

(5)

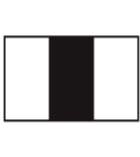

(6)

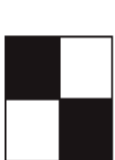

(7)

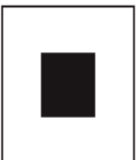

(8)

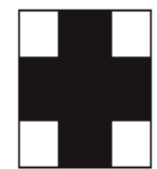

(D)

Figure 2. Generalized Haar-like Features used in our Face Detector. (1-4) Original Haar-like Features used by Viola and Jones, (5-9) Extended Haarlike Features Proposed by Pham and Cham [11]

\subsection{Adaboost for Feature Selection}

AdaBoost is a learning classification function which, when given a set of features and a training set of positive and negative images, learn a classification function any number of machine learning approaches could be used. AdaBoost used to train the classifiers as well as to select a small set of features. When used in its real form, AdaBoost learning function boosts the performance of a simple learning algorithm (sometimes called weak). AdaBoost has the capability to achieve large margins, rapidly that is one of the key features of this algorithm [12].

The method adopts Adaboost as a learning algorithm that constructs a strong classifier composed of weak classifiers. They connected in a cascade. The cascade classifier rejects objects that are apparently false in the early stages, thereby drastically reducing the computation time. Thus, Haar-like feature based Adaboost can effectively learn to construct the best informative weak classifiers from a great number of diverse faces and the convergence of Adaboost also preserved by the robustness.

When the face is detected, the facial features are recognized by using the Adaboost learning algorithm which was originally developed to detect face by Viola [9] [13]. The detector consists in scanning an image by shifting the window at different scales, each sub-window is tested by a classifier made of several stages. The sub-window rejected when it is clearly determined as non-face. However, if it is difficult to discriminate the sub-window, more specific classifier in the late stage determines the sub-window nonface or face.

For instance, positions and sizes of eyes of each diverse upright face are different, but a third type Haar-like feature can cover the region under small variances of faces. It used for the construction of strong classifiers as a linear combination of weak classifiers, as shown in the following equation:

$$
H(x)-\operatorname{sign}\left(\sum_{T-1}^{T} a_{t} h_{t}(x)\right)
$$

Where $h_{t}(x)$ is a weak classifier, Cit is the weight and $H(x)$ is the final strong classifier. In the training process, every weak classifier configured to detect those features that misclassified in previous classifiers [14]. In order to reduce the effect of surrounding light variation in the facial region the histogram equalization is proceeded before applying the Adaboost algorithm for facial feature detection.

\subsection{Attention Cascade Structure}

Cascade classifier method is a face detection algorithm based on Haar-like features. A boosted cascade classifier is very popular for rapid classification. However, the raw 
output of the boosted cascade classifier equilibrated. Although a boosted cascade, classifier is a fast version of Adaboost, the classifier used in limited applications because no one has presented a calibration method for it.

Cascade classifier method is a face detection algorithm based on Haar-like features. The white region's pixel value sum subtracts the black region's pixel value sum can get the characteristic value of feature rectangle, which is used as the face detection's basis [15].

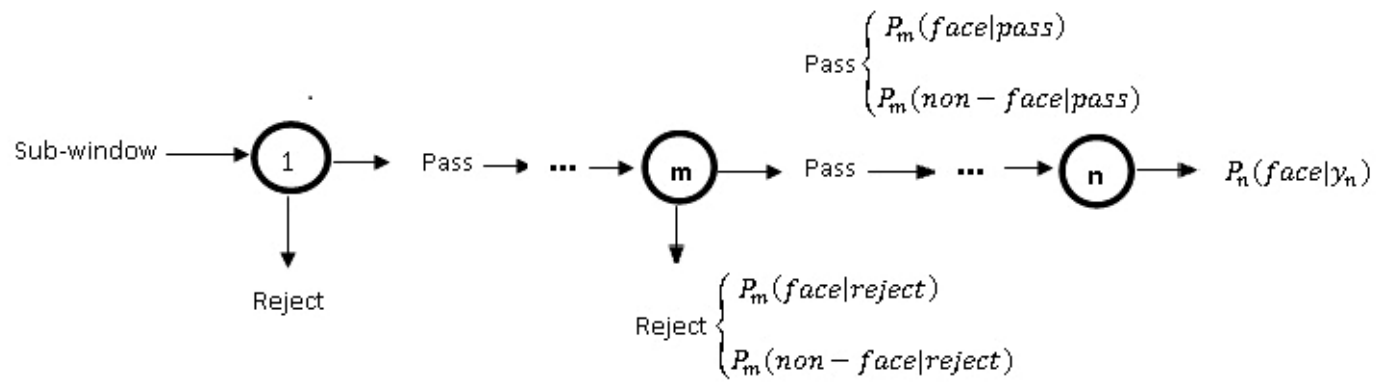

Figure 3. The Process of a Boosted Cascade Classifier: Rejected Sub Windows at Each Stage Subsequently Categorized into Four Groups

As shown in figure 3 shows the classification process of a boosted cascade classifier. At each stage, all sub windows categorized into only four types; rejected faces, rejected non- faces, passed faces, and passed non-faces. It is a critical component in the ViolaJones detector, where the main idea behind it is in building smaller boosted classifiers [16]. Each node is a collection of weak classifiers. Nodes are forming a degenerate decision tree, called a cascade.

The number of classifiers in a node usually increases with level, where later stages have more classifiers, because each node tries to pass all the positive sub-windows to further stages, while still rejecting some of the negative ones. Input sub-window passes a series of nodes, where each node makes a binary decision whether to reject it, or pass it on to the next stage, that way, only a small amount of Sub windows will pass through to the latter stages, with most of the negatives rejected early on, thus vastly improving efficiency. The goal of the proposed calibration method is to obtain a posterior probability using the boosted cascade classifier.

\section{Head Pose Estimation}

The head poses expressed as three angles. Yaw is a rotation angle around an axis from a head top through a neck center. Pitch is a rotation angle around an axis through both ears. Roll is a rotation angle around an axis of the nose through the back of the head. Yao et al. Proposed a method for head pose estimation using an affine motion model of a face [17]. If we assume a face as a plane, then the face motion can express well by the model. The motion model is obtainable with the method described above using two images of a frontal face and the target face. The head angle $\alpha$ is obtained with the equation $\alpha=5(\Theta, 6)$, where $\Theta$ represents the affine motion model and $f$ stands for the focal distance of a camera. If we assume that a circle placed in front of the face and a photograph taken diagonally to the face, then an ellipse projected on the image screen. $F$ is a function to obtain a rotation angle so that a circle projected on the screen. This method can only estimate the rotation angle around the major axis of the ellipse.

We use an algorithm that adopts the affine motion model with a global illumination change $\xi$ [17]. The model can express a motion of the region such as rotation, zoom 
in/out, and transformation. All pixel flow in a region can be express as a set of linear equations. The model comprises seven parameters as presented below.

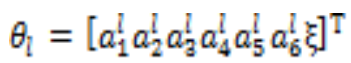

$$
\begin{aligned}
& d_{\theta_{1}}(s)=\left[\begin{array}{l}
u_{1}(x, y) \\
v_{1}(x, y)
\end{array}\right]=\left[\begin{array}{c}
\left.a_{1}^{\mathbb{1}}+a_{2}^{\mathbb{1}} x+a_{2}^{\mathbb{1}} y\right) \\
a_{4}^{\mathbb{1}}+a_{5}^{\mathbb{1}} x+a_{6}^{\mathbb{1}} y
\end{array}\right]
\end{aligned}
$$

Therein, s stands for a coordinate of $(\mathrm{x}, \mathrm{y})$, and $d_{\theta_{\mathrm{I}}}(s)$ Signifies a motion vector at coordinates. The coordinates of the face parts expressed as presented in Figure 4. The nose position is set up as the origin. The coordinates of the other parts defined from the origin. The distance between the face parts normalized so that the coordinates are independent of the image size. For example, they normalized by defining the distance between two eyes as a unit distance. Using these coordinates, angle $\alpha$ can be approximate by the linear equation below.

$$
a=a_{1} x_{r e}+a_{2} y_{r e}+a_{a} x_{\text {le }}+a_{4} y_{\text {le }}+a_{5} x_{m}+a_{6} y_{m}+b
$$

Therein, $\left(x_{r e,} y_{r e}\right),\left(x_{l e,}, y_{l e}\right)$ and $\left(x_{m v} y_{m}\right)$ respectively represent the coordinates of the right eye, left eye, and mouth.
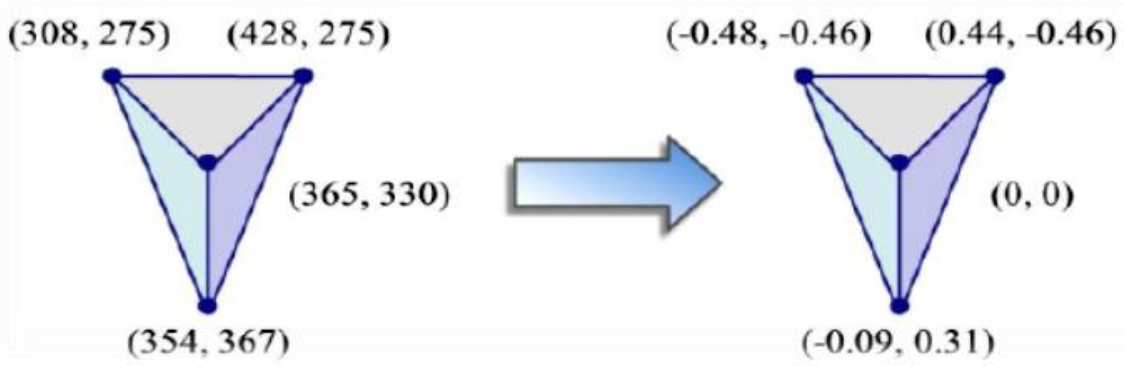

Figure4. Coordinate Expression of face Parts

\section{Experimental Results}

In this section, several experiments carried out to evaluate the performance of our head estimation and face detector. In our propose method, we first demonstrate the superiority of face detection using Haar-like features in terms of the classification efficiency and detection performance. We then show that the Adaboost based feature selection significantly reduces the training time and the number of features required in our system. Finally, we extend our detector to profile faces and discuss how the detector's accuracy affected by poses variations.

We verify the proposed work over three published and widely recognized datasets to address how pose variation affects the performance of our extended multi-view face detector; we test our detector on a set of test images from pointing'04 database [18], ESOGU Face Detection Database [19], and Face Detection Dataset and Benchmark [20]. On all the datasets, the proposed method significantly outperforms the state-of-the-art performances, especially on FDDB. The detection performance of a faster detector with 500 exemplars reported as well to show the proposed approach could have decent performance with a more practical setting. The Results for various Databases are as follows: 


\subsection{Pointing'04 Database:}

We used the Pointing'04 database in this experiment [18]. The head poses database consists of 15 sets of images. Each set contains of 2 series of 93 images of the same person at different poses. There are 15 people in the database, wearing glasses or not and having various skin colors. The pose, or head orientation is determined by 2 angles (h, v), which varies from -90 degrees to +90 degrees. We used data from the database images with the head angle from -60 to $+60 \mathrm{deg}$. First, we manually adjusted the head position for each image in the center roughly. Face part coordinates obtained by application of the proposed algorithm to the sequence.

\section{Results and Discussion:}

An experimental procedure is the following: at first we obtain center coordinates of face parts for a person to learn, learn unknown parameters using the coordinates of facial parts and true angle, then we estimate yaw and pitch angle using the parameters and coordinates of face parts for a person to evaluate, finally we calculate the error between the true angle and the estimated angle.

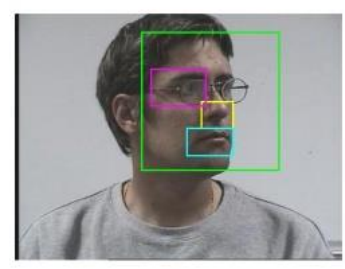

$0-45$

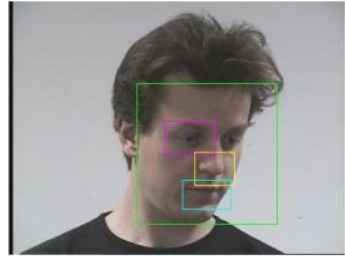

$-30-30$

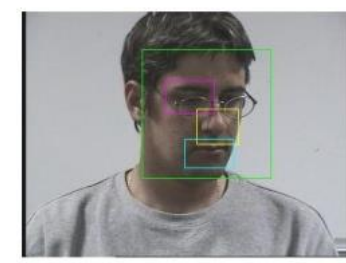

$+30-30$

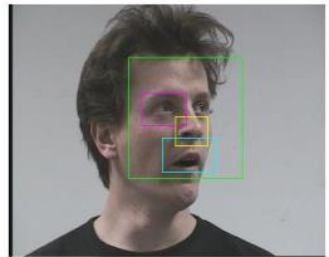

$+15-45$

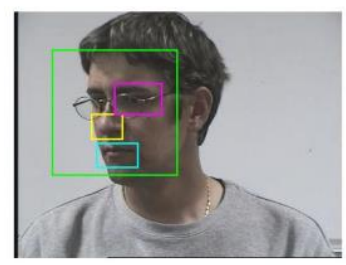

$+15+30$

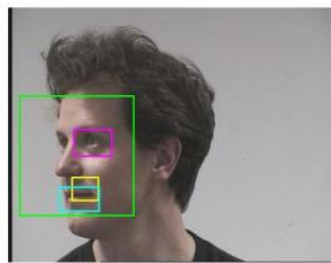

$+30+45$

Figure 5. Three Examples of Two Persons (picked from Pointing '04 Head Pose Image Database) of Poses in which Nose Coordinates are Out of the Circumference Centered around the Nose Location

As shown in the results our proposed method is better than method that based on traditional Haar-Like features [18], the face detection rate of Haar-like method is low. This method can detect just frontal faces. However, profile faces not detected. Much false detection of face parts made. False detection of face parts often occurs because the standard position and size are not considered. It is easy to see that some facial features (in particular, the left/right eye) are not easily detectable. It is worth remarking that ground truth in the Pointing '04 data set is not accurate thus indicated values for the head pose could be more than those reported. The proposed method achieves an even higher rate of right answers than the template method because the proposed method supports shape changes. As shown in Figure 5, face successfully detects a face rotated at an angle between off $-60^{\circ},+60^{\circ}$, we used poses varying from $-45^{\circ}$ to $+45^{\circ}$ because when exceeding this interval, the bilateral symmetry disappears from the image. We tested several configurations, changing the number of classes each time. 


\subsection{ESOGU Face Detection Database}

Where the ESOGU face detects from the Eskisehir OsmanGazi University, The original database contained 285 higher-resolution color face images, including faces that appear in a wide range of image positions and scales, also complex backgrounds, occlusions, illumination variations and multi-pose head images. We extended the database by adding 382 new images. Thus, the database now includes 667 images that contain 2042 annotated frontal faces.
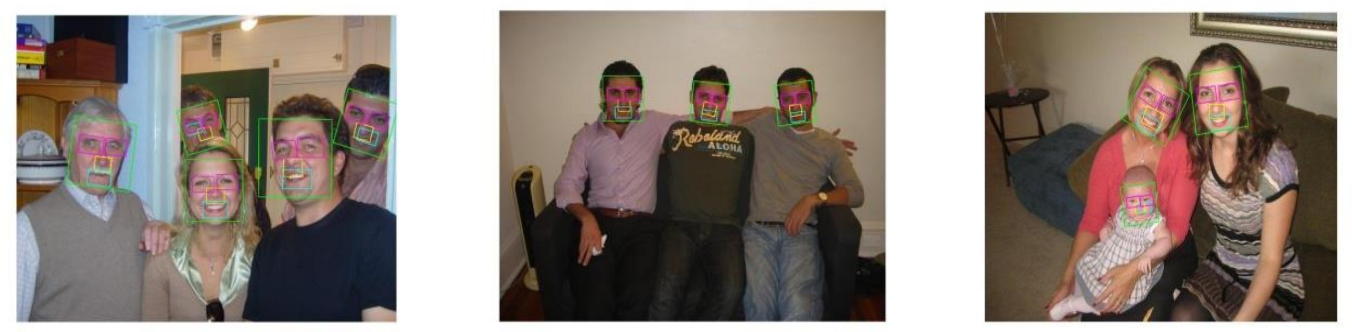

\section{Figure 6. Some Examples of the Output of our Method from the ESOGU Face Dataset}

\section{Results and Discussion}

Of many faces, the sizes of the images are too small to search for the parts, thus we up sample the facial region in such cases. However, returned parts mostly fail for up sampled low resolution faces. To this end, we visually counted the number of faces returned by the people's tagging tool of this system. We considered all returned face images, as true positives although some faces do not satisfy PASCAL VOC overlapping criterion. We also ignored all false positives coming from the background [19]. Most of those images exhibit a very large variety in pose, lighting, expression as well as general imaging conditions, Many images exhibit partial occlusions that caused by head pose, objects (e.g., glasses, scarf, and food), body parts (hair, hands) and shadows.

\subsection{Face Detection Dataset and Benchmark (FDDB)}

FDDB [20] contains 5,171 annotated faces in 2,845 images. The images in this dataset extracted from news articles those present large face appearance variations. The evaluation procedure is standardized and researchers expected to use the same evaluation program to report the results. FDDB is a challenging face detection benchmark designed to study unconstrained face detection.
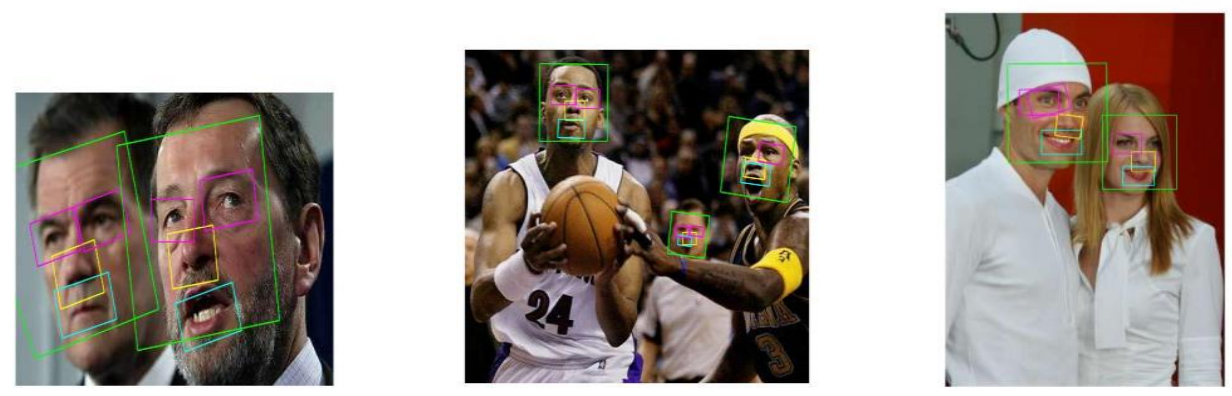

Figure 7. Some Examples of from FDDB Data Set, when the Green Rectangles Show True Human Annotations Correct Detections, and Pink Rectangles Show the Eye, Yellow Rectangle Shows the Nose and Blue Rectangle Show the Mouth 


\section{Results and Discussion}

The combination of detection and tracking enables efficient detection only around the search area limited by the tracking. It also reduces false detection because of processing continuity between two frames. Because of the combination, a profile face can estimate accurately. In addition, the method re-initializes the position and size of the face and face parts in every frame. The initialization corrects tracking jitter immediately. Furthermore, undetected face revised in terms of position and size using detected face parts.

Experimental conditions are Intel Core i3-2350M CPU, $2.30 \mathrm{GHz}, 2 \mathrm{G}$ RAM, Windows 7, and MATLAB2012a version. Our tests on challenging data sets show that the current face detectors mostly tolerate to small rotations and illumination changes, and, successful to detect faces when the rotations and illumination changes are severe. They also typically can detect faces smaller than 30x30 pixels. Part-based models work well if the face images are large eyes and mouth are clearly visible within the face region. Results also show that there is not a significant gap between the performances of the best face detectors introduced by the academic community and commercial face detectors. The results are encouraging, and both face and landmark detectors can be integrated into face recognition systems without hesitation. In addition, results on current face recognition databases are mostly saturated, and it is necessary to introduce more challenging face recognition databases.

\section{Conclusion}

Visual estimation of head pose is desirable for computer vision applications such as face recognition, human computer interaction, and affective computing. However, accurate estimation of head poses in an uncontrolled environment is still a grand challenge. This paper proposes a novel feature representation model for accurate pose estimation. In this paper, we presented a novel, general-purpose, geometrical model for head pose estimation, based on the detection of facial features. We address the problem of head pose estimation from a facial in RGB images, the combination of face detection using propose Haar-like feature method to detect people's faces, noses, eyes, mouth, and tracking by the affine motion model estimation enables detection of a profile face. $\mathrm{We}$ proposed head-pose estimation using the cascade object detector, we present an efficient boosted exemplar based face detector utilizing exemplar-based weak detector.

The head poses easily estimated by obtaining the information of nose and eyes from facial features. The constraints in estimating head pose in previous works such as using stereo vision or using an initial template from frontal face, etc. are not necessary in the proposed head pose estimation technique. It also efficiently used in real-time fashion for a sequence of data set with less computational cost. This data set includes faces under a wide range of conditions including illumination, scale, and pose and camera variation. Nevertheless the system which work under this algorithm are subjected to the same set of conditions and but the algorithm is flexible enough to adjust according to the changing conditions. Detailed comparisons with the other methods remain as a subject for future work.

\section{Acknowledgements}

This research is supported by the National Science Foundation of China under Grant No. 60970098, Modelling for Human Visual System and its application; The National Science Foundation of China under Grant No. 61173122, A Fast Three-dimensional Reconstruction Based on Image by Simulating Mechanisms of Human Visual System. 


\section{References}

[1] G. L. Marcialis, F. Roli and G. Fadda, "A novel method for head pose estimation based on the Vitruvian Man”, International Journal of Machine Learning and Cybernetics, vol. 5, (2014).

[2] B. Czupryński, A. Strupczewski, "High Accuracy Head Pose Tracking Survey", Active Media Technology, (2014).

[3] B. O. Han, Y. N. Chae, Y.-H. Seo and H. S. Yang, "Head Pose Estimation Based on Image Abstraction for Multiclass Classification", Information Technology Convergence, (2013).

[4] T. Vatahska, M. Bennewitz, S. Behnke,"Feature-based head pose estimation from images", 7th IEEERAS International Conference on Humanoid Robots, (2007); Pittsburgh, PA.

[5] J. Whitehill and J.R. Movellan, "A discriminative approach to frame-by-frame head pose tracking", 8th IEEE International Conference on Automatic Face and Gesture Recognition, (2008); Amsterdam.

[6] L. Morency, J. Whitehill and J. Movellan,"Generalized adaptive view-based appearance model Integrated framework for monocular head pose estimation", 8th IEEE International Conference on Automatic Face and Gesture Recognition, (2008); Amsterdam.

[7] B. O. Han, S. Lee and H. S. Yang, "Head pose estimation using image abstraction and local directional quaternary patterns for multiclass classification", pattern Recognition Letters, vol. 45, (2014).

[8] A. Gee and R. Cipolla, "Determining the gaze of faces in images", Image and Vision Computing, (1994).

[9] P. Viola, M. Jones, "Rapid object detection using a boosted cascade of simple features", Proceedings of CVPR 2001 IEEE International Conference on Computer Vision and Pattern, (2001); Kauai, HI, USA.

[10] W. Kim, H. Lee, W. Lee, H. Song, L. Chun, "A Feasible Face Pose Estimation by Evaluating 3D Facial Feature Vectors from 2D Features", Advanced Communication Technology (ICACT), 2013 15th International Conference,(2013); PyeongChang.

[11] M.T. Pham and T.J. Cham, "Fast training and selection and Haar features using statistics in boostingbased face detection", in: Proceedings of ICCV $200711^{\text {th }}$ IEEE International Conference on Computer Vision, (2007), 14-21 Oct, Rio de Janeiro.

[12] Jatin Chatrath, Pankaj Gupta, Puneet Ahuja , Aryan Goel, Shaifali M.Arora ,"Real Time Human Face Detection And Tracking", International Conference on Signal Processing and Integrated Networks ,(2014);Spin.

[13] P. Viola and M. Jones, "Robust real-time face detection", International journal of computer vision, vol. 57, no. 2, (2004), pp. 137-154.

[14] H. Leventi, C. Livada, I. Gali, “Towards Fixed Facial Features Face Recognition”, 21 International Conference on Systems, Signals and Image Processing, (2014); Dubrovnik.

[15] R. Meng, Z. Shengbing, L. Yi, Z. Meng, "CUDA-based Real-time Face Recognition System”, Digital Information and Communication Technology and it's Applications (DICTAP), 2014 Fourth International Conference on,(2014); Bangkok.

[16] J. Yan, X. Zhang, Z. Lei and S.Z. Li, "Real-time high performance deformable model for face detection in the wild", International Conference on Biometric, IEEE, (2013), 4-7 June, Madrid.

[17] M. Mitama and Y. Matsuda, "Integrated Face Detection Tracking, and Pose Estimation", Signal Processing (ICSP), 2012 IEEE 11th International Conference on, (2012); Beijing.

[18] N. Gourier, D. Hall, J. L. Crowley, "Estimating Face Orientation from Robust Detection of Salient Facial Features", Proceedings of Pointing ICPR, International Workshop on Visual Observation of Deictic Gestures, (2004); Cambridge, UK

[19] http://mlcvdb.ogu.edu.tr/facedetection.html

[20] V. Jain, E. Learned-Miller, Fddb,"A benchmark for face detection in unconstrained settings", University of Massachusetts, (2010).

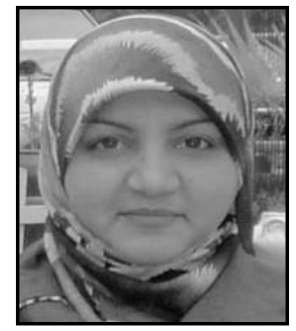

Hiyam Hatem, received the BS degree from the department of computer science, Baghdad University, Iraq, 2003. The master degree from Huazhong University of Science and Technology Wuhan, China, in 2010.Currently, she is a PhD student in School of information science and Engineering at Central south university, Changsha, China. Her research interests include face processing and recognition, object detection, pattern recognition, computer vision, and biometrics. 

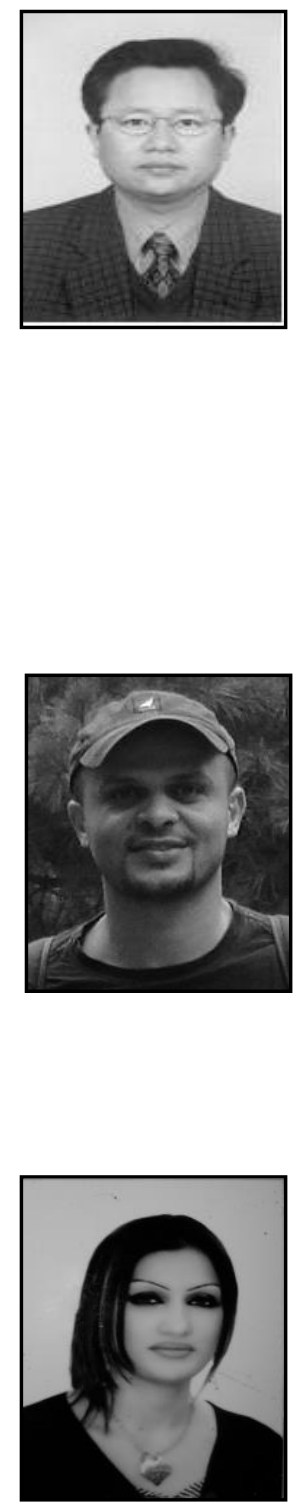

Jumana Waleed is a Ph.D. student in the School of information science and Engineering at Central South University, Changsha, China. Her research activity focuses on image processing, and information security working on digital watermarking. She received the B.S. degree in computers sciences from the Al-Yarmouk University College, Iraq, in 2004, and the M.S. degree in Computer Science/Data Security from the University of Technology, Baghdad, Iraq, in 2009.

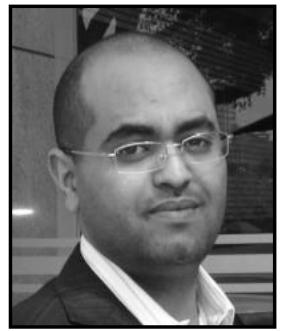

Mohammed Lutf : Ph.D. student in the Department of Electronics and Information Engineering at Huazhong University of Science and Technology, Wuhan ,China.. He is an active researcher in the area of Arabic character and handwriting recognition. He received his B.S. Degree in Telecommunication Engineering from Dalian Maritime University, Dalian, China, in 2005 and an M.S .Degree in Communication and Information Systems from Huazhong University of Science and Technology, Wuhan, China, in 2010. 
International Journal of Multimedia and Ubiquitous Engineering

Vol. 10, No. 3 (2015) 